\title{
A Comprehensive Study of Dispute Resolution using Artificial Neural Network in Build Operate and Transfer (BOT) Project
}

\author{
Asra Fatima ${ }^{1}$ \\ Research Scholar, Department of Civil Engineering \\ Gandhi Institute of Technology and Management (GITAM University), Hyderabad, India. \\ E-mail: asra.fatima03@gmail.com \\ B.S.R.K Prasad ${ }^{2}$ \\ Head of the Department and Professor, Department of Civil Engineering \\ Gandhi Institute of Technology and Management (GITAM University), Hyderabad, India. \\ E-mail: hodcivil_hydcampus@gitam.in \\ Seshadri Sekhar T. ${ }^{3}$ \\ Dean and Professor, Project Guide, Department of Civil Engineering, \\ National Institute of Construction Management and Research (NICMAR), Hyderabad, India. \\ E-mail: ss.tirumala@gmail.com \\ ${ }^{3}$ Orcid: 0000-0003-4037-9396 \& Scopus Author ID: 35491505500
}

\begin{abstract}
BOT projects plays $\mathrm{s}$ vital role in development of infrastructure of the country. A BOT projects involves many parties were dispute can probably occur, which when not resolved on time becomes very expensive in terms of finances, personnel, time, and opportunity costs. In order to minimize dispute data is collected from various construction firms with the help of questionnaire survey, which consist of 5 point scale (1-5, 1-very poor influence to 5 very strong influence) were respondents are requested to rate the factors with their degree of involvement in the arousal of disputes. Analysis shall be done by using SPSS software for identification of 20 factors and then developing neural networks model in resolving disputes. In doing so identified factors influencing the arousal of disputes will be fed to artificial neural network and a network building will be done. Various iterations shall help to assess the effect of change in network parameters. Comparison of their results made in the study gives an optimum combination of parameters for effective resolution of disputes using neural network.
\end{abstract}

Keywords: Built operate and transfer, dispute resolution, Artificial neural network

\section{INTRODUCTION}

The Construction industry is dynamic and competitive environment. Relationship within construction, between clients, contractors, sub-contractors and suppliers, are often adversarial. The risks associated with construction projects can be high, the process is complex and obligations are often onerous. Dispute resolution in the construction industry has been topical because of the growing concern over the ever increase in management resources to deal with construction disputes. Innovative dispute resolution processes, generically described as alternative dispute resolution, have been developed and introduced to facilitate amicable resolution. ADR is a non adversarial technique which is aimed at resolving disputes without resorting to the traditional forms of either litigation or arbitration. The use of Artificial Neural Network (ANN) in dispute resolution is extending this trend at the double, by contributing to a more efficient use of ADR methods. The success or otherwise of a resolution process depends on a wide range of factor

\section{NECESSITY OF THE STUDY}

The Construction industry is dynamic and competitive environment. Relationship within construction, between clients, contractors, sub-contractors and suppliers, are often adversarial. The risks associated with construction projects can be high, the process is complex and obligations are often onerous. The way demand is put on the industry through competitive tendering procedures can often increase adversity. It is often said that in such commercial atmosphere conflict is inevitable; indeed conflict is a necessary part of the competition of commercialism.

Disputes are a common occurrence within the construction industry. The costs associated with resolving disputes are often high, and the time involved can be very long. Construction disputes, when not resolved in a timely manner, become very expensive in terms of finances, personnel, time, and opportunity costs. The visible expenses (e.g., attorneys, expert witnesses, the dispute resolution process itself) alone are significant. The less visible costs (e.g., company resources assigned to the dispute, lost business opportunities) and the intangible costs (e.g., damage to business relationships, potential value lost due to inefficient dispute resolution) are also considerable, although difficult or impossible to quantify. 
Respected professionals estimate that construction litigation expenditures in the United States have increased at an average rate of 10 percent per year over the last decade, and now total nearly $\$ 5$ billion annually, and in India cost is around 50000 to 1 lakh. The factors which are responsible for a dispute to occur is identified and the measures which are to be taken in order to minimize it is also mentioned.

\section{LITERATURE REVIEW}

Sia on cheung and H.Y .Pang (2014) stated that the construction disputes are widely considered as inevitable. The supporting evidence of this general belief is largely anecdotal. An empirical test of this proposition is required. The conceptual framework of construction disputes is further developed and a, fault tree methodology is used to operate the framework whereby the inter-relationships among dispute artifacts are expressed in logic gates. This concept displays the logic relationships and further allows assessment of occurrence likelihood. In view of the imprecise nature, fuzzy sets occurrence likelihood assessments of the artifacts are used. The findings support that construction disputes are inevitable if no conscious effort is made to minimize its occurrence.

Philip S. LaBarre1 and Islam H. El-Adaway (2014) aimed that the construction industry significantly contributes to the U.S. economy. However, this important industry is negatively affected with many challenges including safety issues, design errors, delay, and changes. To this end, performance measurement has become a commonly used tool for evaluating processes, managing human resources, and formulating corrective strategies. One of the most important aspects of performance measurement is the ability to perform benchmarking. Using case studies of projects carried out by the U.S. Army Corps of Engineers (USACE) in its Vicksburg District, this paper investigates how benchmarking can be used to evaluate and measure performance of the associated contractors, and thus mitigate conflicts, claims, and disputes. The utilized methodology encompassed three interdependent steps in which the writers: (1) collected project data for 40 contractors who performed work for USACE using file reviews for submittals, requests for information (RFIs), claims, progress reports, and internal evaluation forms by the associated senior project managers; (2) defined the associated performance measures; and (3) analyzed the results to identify areas in which contractors needed to improve performance. The writers utilized five performance measures including quality control, timely performance, and effectiveness of management, compliance with labor standards, and compliance with safety standards. These measures were applied on a five-point rating system and categorized by using three characteristic groups, including use of subcontractors, contract modifications, and final contract price. It was shown that contractors who had higher performance measures did actually have fewer contractual and legal problems-either during construction or after completion - than those with lower ratings. Also, contractors with an outstanding rating in one area never had a contractual and legal problem in that area. This research supports the effectiveness of benchmarking as a tool to promote changes within the construction industry based on knowing strengths and weaknesses. The findings should better help contractors and owners complete their projects on time, more safely, and with fewer errors and changes

Mohammed Al Qady et al. (2013) stated that Dispute resolution procedures specified in construction contracts commonly contain conditions precedent that can ultimately affect the entitlements of the parties in case a dispute occurs. This study discusses various aspects related to conditions precedent in dispute resolution provisions, namely, notifications of claims as a condition precedent, architect/engineer (A/E) decision as a condition precedent, mediation as a condition precedent, timely submissions as a condition precedent, language for establishing conditions precedent, waiver of conditions precedent by courts, and the authority to issue such waiver. Legal decisions related to each topic are discussed, and general guidelines for dealing with conditions precedent in dispute resolution procedures are given.

K.N. Jha and C.T. Chockalingam (2009) described that project manager always encounters difficulties in predicting the performance of a construction project. Thus, there is a need to identify the predictor variables used to predict the performance of the construction project. The 11 success factors derived earlier have been revisited. Out of these factors, project performance predictors have been identified using artificial neural network. Literature pertaining to performance prediction models has been reviewed and the superiority of ANN in performance prediction is established. Various steps in the ANN applications are clearly explained. The performance prediction models have been derived for all the four project performance criteria: schedule, cost, quality, and no-dispute. The steps to develop a user-interactive model to predict the performance of the construction project based on ANN are also explained. The prediction models may prove to be helpful to the project manager, project team, and top management to predict the performance of the project during its course.

\section{RESEARCH METHODOLOGY}

The methodologies to achieve the objectives of identification of factors which can be used to predict the dispute occurrence in construction projects and development of prediction model is considered in Figure 1 which represents research methodology as a flow chart.

For determination of factors affecting the arousal of dispute data is collected using First stage questionnaire survey and analyzed by SPSS Software. After application into software 
the factors for each criterion (i.e cost, and duration) from the identified factors shall be assessed. The analyses result in a number of common factors among them, while a few other factors emerged predominantly in only one or more criterion.

The union of all common and uncommon factors shall be considered. And 20 factors will be identified from first stage questionnaire survey; later second stage questionnaire survey will be conducted to understand the impact of the 20 factors on project performance and a ANN model will developed to predict the key factors responsible for arousal of dispute. Below is Figure 1 which represents the research methodology.

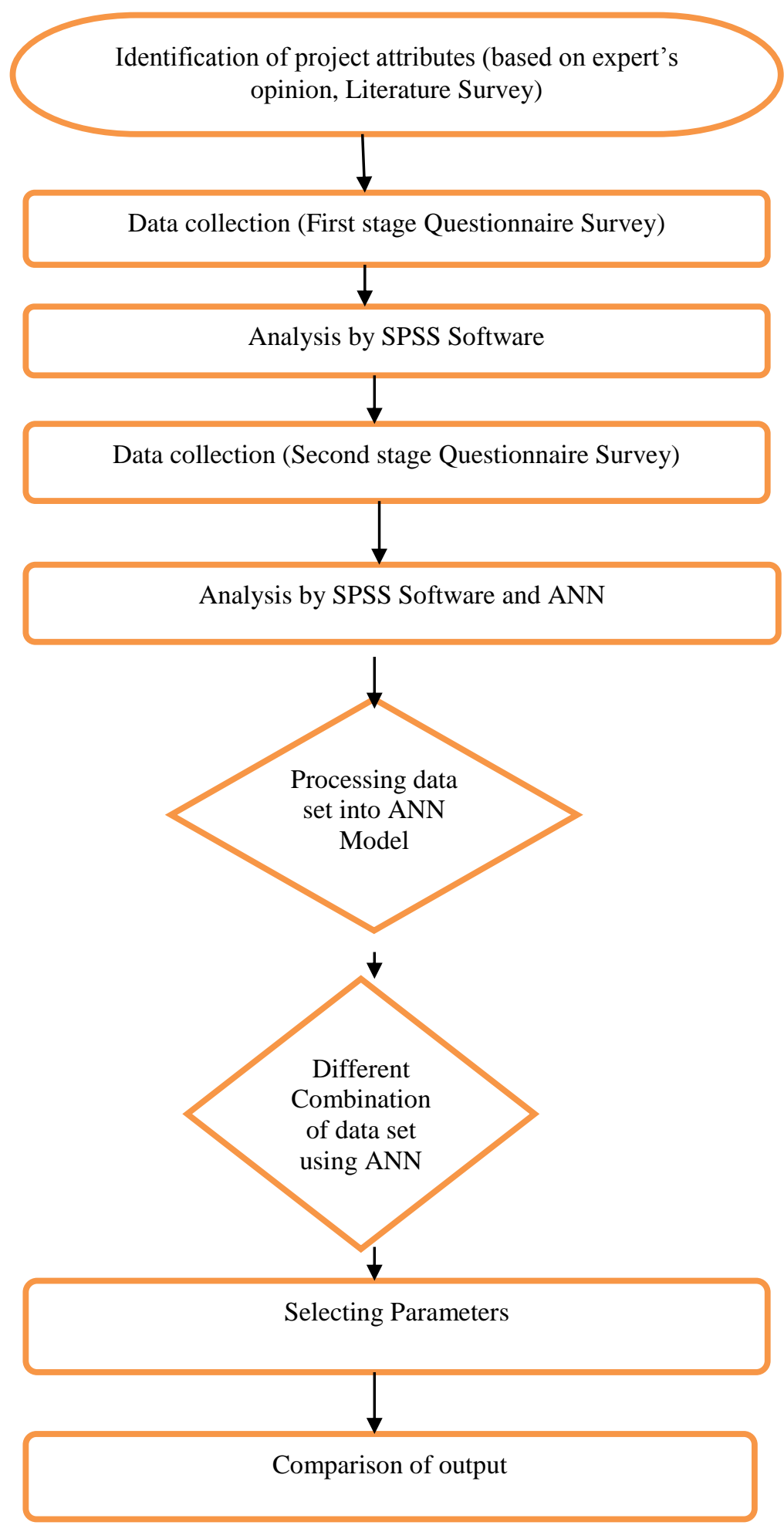

Figure 1: Model Development of ANN 


\section{PREDICTION MODEL}

To ensure that these factors are the key determinants that affect arousal of dispute and also to develop the dispute prediction model for the same, ANN methodology was employed. ANN modeling was chosen because of its robustness, ability to adapt to unknown data sets, and good learning capability. ANN has been adopted to investigate the factors that affect arousal of dispute in contruction projects in Hongkong (Sia on cheung et al., 2000). In the subsequent sections, the details of the proposed prediction model are given. An ANN is an information processing system that displays a similar behavior to that of its biological analog. It is essentially a mathematical model in which the information processing occurs in a number of simple elements called neurons (nodes), and the signals are transmitted between neurons over connection links that has an associated weight with it. Each neuron applies an activation function (transfer function) to the incoming signal to determine its output signal (Zurada, 1992). McCulloch and Pitts proposed a binary threshold unit as a computational model for an artificial neuron as shown in Figure 2. This mathematical model computes a weighted sum of its $n$ input signals $\mathrm{Xj} 1 / 41,2$ up to $\mathrm{n}$ and generates an output of 1 , if it is above a certain threshold or else gives an output of 0 (referred in Zurada, 1992).

The ANN models use various activation functions such as linear, sigmoid, and Gaussian functions out of which the sigmoid is the most commonly used function (Jain et al., 1996).

To express mathematically, each neuron $\mathrm{j}$ sums its weighted input as follows:

$$
\text { net }_{j}=\sum_{j=1}^{n} w_{j} x_{j}
$$

The output of a neuron, $y$ is a function of its weighted input, expressed as follows:

$$
y=f\left(\text { net }_{j}\right)
$$

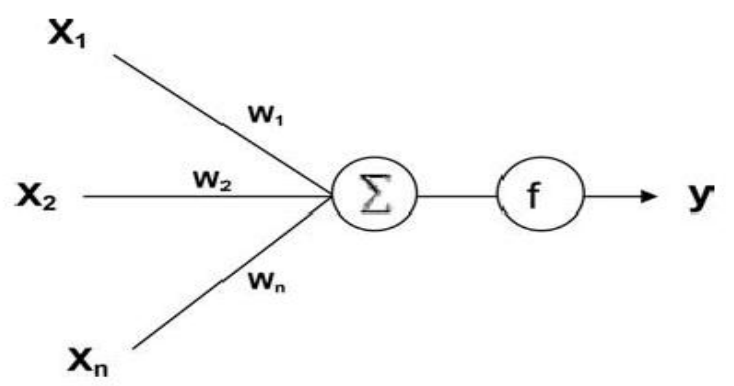

Figure 2: Prediction model of ANN

\section{NETWORK STRUCTURE}

Feed forward neural network architecture has been chosen for constructing the ANN model. It consists of an input layer, output layer and hidden layers if required. The input layer provides input data to the network, and the size of the input layer, or the number of neurons (nodes) which are correlated factors for the dispute arousal criterion in this case. When the hidden layers are used, the number of nodes in the hidden layer is decided by trial and error. The hidden nodes on receiving the values of the inputs calculates the weighted sum of the inputs and according to the transfer function squashes the values to a limited range (Edwards, 2007). The resulting values are then fed as input to the output nodes which again performs the same function. The output layer in this case is the dispute occurrence factors. Table II gives the description of the sigmoid and linear transfer functions used in MATLAB software.

\section{TRAINING THE MODEL}

The main aim of neural network training is to minimize the output error by adjusting network weights and biases. Several learning algorithms have been developed for ANN. According to Jain et al. (1996) back propagation learning algorithm with feed forward network architecture is most suited for predictions. Back propagation is a learning algorithm for a feed forward network which is a supervised learning process based on error correction learning rule. In back propagation, input and the corresponding output are used to train a network until it can approximate a function and associate input with specific output. Properly trained back propagation networks gives reasonable answers when presented with new inputs in most cases.

The inputs are sent forward and then the errors are propagated backwards. The training normally starts with random weights and biases, which are adjusted by the algorithm for minimizing errors. Standard back propagation in MATLAB is gradient descent algorithm. Other important algorithms in MATLAB are Levenberg-Marquardt, Conjugate gradient algorithm, resilient back propagation algorithm etc. In general no single algorithm suits all applications, the usual strategy is to experiment with these algorithms and find the most suitable one for the given application.

\section{CONFIGURATION OF ANN}

Once the above details are defined, the input-output data is divided into training and validation data sets. There are two methods available for training and testing the models - the hold out method and a re-sampling method called random sub-sampling (Edwards, 2007). In case of the hold out method, the data sets are split into two - usually $2 / 3$ for designing (training set) and $1 / 3$ for the estimating the true performance (testing and validation set). While in the latter, 
multiple random train and test experiments are performed on training and testing samples.

Table 1: Configuration of ANN

\begin{tabular}{llll}
\hline Transfer function & Input range & Output range & Function \\
\hline Log sigmoid & Plus and minus infinity & 0 and 1 & $f(x)=\frac{1}{1=e^{-x}}$ \\
Hyperbolic tangent sigmoid & Plus and minus infinity & -1 and +1 & $f(x)=\frac{e^{x}-e^{-x}}{e^{x}+e^{-x}}$ \\
Linear function & Plus and minus infinity & $\begin{array}{l}\text { Plus and minus } \\
\text { infinity }\end{array}$ & $f(x)=x$ \\
\hline
\end{tabular}

According to Edwards (2007) and Goh (1995) when the number of data sets is sufficient the hold out method proves to be efficient. Hence, about two-thirds of the data are used for training and the rest for testing and validation.

\section{CONCLUSION}

As BOT project involves many parties, including the government, promoter, construction contractor, operating firms, financers and other parties were dispute are likely to occur, which when not resolved in a timely manner becomes very expensive in terms of finances, personnel, time, and opportunity costs. Data collected from the respondents are requested to rate the factors affecting dispute with their degree of involvement in the arousal of disputes. Analysis is done by using SPSS software will help in identification of 20 factors which will be then fed into neural network model in resolving disputes. Later second stage questionnaire will be used to find the impact of these parameters on project completion which in turn helps in identification of key factors which can be avoided in any BOT projects.

\section{REFERENCE}

[1] Asra Fatima, Bellam Sivarama Krishna Prasad, T. Sheshadri Sekhar and Mannan Hussain (2017), Application Of Neural Network For Resolution Of Dispute In Built Operate And Transfer (Bot) Project: A Review,I-Manager's Journal On Civil Engineering, 7 (2): 34-40.

[2] N. B. Chaphalkar and Sayali S. Sandbhor, (2015), Application of Neural Networks in Resolution of Disputes for Escalation Clause Using Neuro-Solutions, KSCE Journal of Civil Engineering, 19(1):10-16.

[3] Jui-Sheng Chou1 and Chieh Lin (2013), Predicting Disputes in Public-Private Partnership Projects: Classification and Ensemble Models, J. Computing in Civil Engineering , ASCE., 27:51-60.

[4] Xinyi Song, S.M.ASCE, Feniosky Peña-Mora, M.ASCE,Carol C. Menassa, M.ASCE and Carlos A.
Arboleda (2012), Insurance as a Risk Management Tool for ADRImplementation in Construction Disputes, $J$. Constr.Eng. Manage. ASCE, 138, 14-21.

[5] S. O. Cheung, C. M. Tam, and F. C. Harris (2000), Project dispute resolution satisfaction classification through neural network." J. Manage. Eng., ASCE, 16, 7079.

[6] Edwards, S.R. (2007), Modelling perceptions of building quality - a neural network approach, ,Building and Environment, 42, 2762-77.

[7] SPSS 16.0 (2008), SPSS Inc., Chicago, IL, available at: www.spss.com (accessed 16 October 2008).

[8] Jain, A.K., Mao, J. and Mohiuddin, K.M. (1996), Artificial neural networks: a tutorial, IEEE, 29 ( 3), 3144.

[9] Jha, K.N. (2004), Factors for the success of a construction project: an empirical study, doctoral thesis, Indian Institute of Technology, Delhi 